\title{
Fabrication of Complex Three-Dimensional Nanostructures from Self-Assembling Block Copolymer Materials on Two-Dimensional Chemically Patterned Templates with Mismatched Symmetry
}

\author{
Kostas Ch. Daoulas and Marcus Müller* \\ Institut für Theoretische Physik, Georg-August Universität, 37077 Göttingen, Germany \\ and Department of Physics, University of Wisconsin-Madison, Madison, Wisconsin 53706-1390, USA \\ Mark P. Stoykovich, Sang-Min Park, Yioryos J. Papakonstantopoulos, Juan J. de Pablo, and Paul F. Nealey ${ }^{\dagger}$ \\ Department of Chemical and Biological Engineering, University of Wisconsin-Madison, Madison, Wisconsin 53706-1691, USA \\ Harun H. Solak \\ Laboratory for Micro- and Nanotechnology, Paul Scherrer Institut, CH 5232 Villigen PSI, Switzerland
}

(Received 21 August 2005; published 24 January 2006)

\begin{abstract}
A study is presented of the self-assembly of a lamella-forming blend of a diblock copolymer and its respective homopolymers on periodically patterned substrates consisting of square arrays of spots, that preferentially attract one component, as a function of pattern dimensions and film thickness. The blend morphology follows the pattern at the substrate and forms a single quadratically perforated lamella (QPL). At intermediate film thicknesses necks connect this QPL to the film surface, resulting in a bicontinuous morphology. The necks do not register with the underlying square lattice but exhibit a substantial amount of hexagonal short-range order. For thicker films we observe bicontinuous morphologies consisting of parallel lamellae with disordered perforations. These results demonstrate a promising strategy for the fabrication of complex interfacial nanostructures from two-dimensional chemically patterned templates.
\end{abstract}

DOI: 10.1103/PhysRevLett.96.036104

The self-assembly of copolymer materials in thin films is determined by an intricate interplay between interfacial interactions [1,2], breaking of translational symmetry, and structural frustration due to the incompatibility between the natural periodicity of the bulk structure and film thickness $[3,4]$. Confinement and surface effects can result in morphologies that are absent in the bulk, e.g., surface reconstructions [5].

Chemically patterned substrates can direct the assembly of adsorbed layers or thin films of block copolymers [613]. Recent experiments have demonstrated that by matching the symmetry and length scale of the substrate pattern with the bulk morphology, it is possible to achieve defectfree replication of the substrate pattern [13-15]. Exciting opportunities exist to extend this general strategy for the fabrication of complex three-dimensional (3D) structures by using self-assembling materials in conjunction with templated substrates.

Here we demonstrate how thin films of block copolymer materials that form lamellae in the bulk can be directed to assemble on chemically patterned substrates consisting of square arrays of spots to form a disordered, bicontinuous morphology. This disordered morphology results from the interplay between the periodic morphology of the bulk material and the two-dimensional (2D) periodic substrate pattern which differ in symmetry and length scale. A similar interplay has been discussed for physisorbed monolayers on periodically corrugated substrates [16]. The surface reconstruction on patterned substrates observed here,
PACS numbers: 81.07.-b, 61.25.Hq, 61.30.Hn, 81.16.Fg

however, imparts a complex structure in the third direction, perpendicular to the substrate, propagating through the entire film thickness.

A polystyrene (PS) brush was grafted to a silicon substrate and subsequently coated with photoresist. Fourbeam extreme ultraviolet interference lithography $[17,18]$ was used to pattern a square array of spots with nearest neighbor center-to-center distances of $71 \mathrm{~nm} \leq \lambda \leq$ $113 \mathrm{~nm}$ and a range of spot radii, $R$. Treatment of the sample with an oxygen plasma chemically modified the PS brush not masked by photoresist to be oxygen-rich and highly polar. Removal of the remaining photoresist resulted in the desired chemically patterned substrate. A $44 \mathrm{~nm}$ film of a symmetric ternary blend [19], consisting of $60 \mathrm{wt} \%$ poly(styrene- $b$-methyl methacrylate) (PS- $b$-PMMA, $\quad \mathrm{Mn}=104 \mathrm{~kg} / \mathrm{mol}), \quad 20 \quad$ wt $\% \quad$ PS (40 kg/mol), and $20 \mathrm{wt} \%$ poly(methyl methacrylate) (PMMA, $41 \mathrm{~kg} / \mathrm{mol}$ ), was deposited on the patterned substrate and annealed at $190^{\circ} \mathrm{C}$ for 7 days. On uniform substrates this blend orders into a lamellar phase with a periodicity of $L_{0}=70 \mathrm{~nm}$ [15]. On the patterned substrate the PS domains preferentially wet the chemically unmodified regions of the PS brush, while the PMMA domains preferentially wet the oxygen plasma treated spots. Scanning electron microscopy (SEM) and atomic force microscopy (AFM) were used to examine the top surface of the thin films.

Single chain in mean field (SCMF) simulations [20] provide additional information about the $3 \mathrm{D}$ structure of the 
ternary blend and allow for a systematic exploration of parameters, e.g., surface pattern dimensions and film thickness. This particle-based self-consistent field (SCF) method simulates a large ensemble of single chains in external fields that, in turn, depend on the instantaneous density distribution created by the ensemble. In the limit of an infinitely large ensemble the method reproduces the results of equilibrium SCF theory [21]. For finite ensembles characterized by $\overline{\mathcal{N}} \equiv\left(\Phi R_{e}^{3}\right)^{2}$ ( $\Phi$ being the polymer chain number density and $R_{e}$ the diblock's end-to-end distance), the simulations capture some fluctuations. $\overline{\mathcal{N}}=16384$ matches the experiment. Comparing the bulk lamellar spacing, $L_{0}=2.26 R_{e}$, with the experiment we identify the length scale. Fields are discretized in real space with resolution $R_{e} / 6$. Chains are represented by a discretized Edwards Hamiltonian with $N=15+17$ segments for a diblock copolymer, and $N_{\mathrm{PS}}=12$ and $N_{\mathrm{PMMA}}=13$ for homopolymers. The interaction free energy takes the form: $\frac{\mathcal{F}_{\mathrm{SCF}}}{k_{\mathrm{B}} T \Phi}=\int \mathrm{d}^{3} \mathbf{r}\left(\chi N \hat{\phi}_{\mathrm{PS}} \hat{\phi}_{\mathrm{PMMA}}+\frac{\kappa N}{2}\left[\hat{\phi}_{\mathrm{PS}}+\hat{\phi}_{\mathrm{PMMA}}-1\right]^{2}+\right.$ $\left.U_{\mathrm{W}}(\mathbf{r})\left[\hat{\phi}_{\mathrm{PS}}-\hat{\phi}_{\mathrm{PMMA}}\right]\right)$, where $\hat{\phi}_{\mathrm{PS}}(\mathbf{r})$ and $\hat{\phi}_{\mathrm{PMMA}}(\mathbf{r}) \mathrm{de}-$ note the microscopic volume fractions. The field acting on PS-segments is given by $W_{\mathrm{PS}}(\mathbf{r})=\Phi \delta \mathcal{F}_{\mathrm{SCF}} / \delta \hat{\phi}_{\mathrm{PS}}(\mathbf{r})$. We use $\chi N=36.7$ for the Flory-Huggins parameter $[22,23]$ and $\kappa N=50$. Morphologies were obtained after a quench from the disordered phase [24]. The polymer segment-substrate interactions are short-ranged in the direction, $x$, normal to the substrate: $U_{\mathrm{W}}(x, y, z)=\frac{\Lambda N f(y, z)}{0.15 R_{e}} \times$ $\exp \left[-\frac{x^{2} / 2}{\left(0.15 R_{e}\right)^{2}}\right]$ with $\Lambda N=3 . f(y, z)$ adopts the values 1 or $-f_{g}$ describing the square array of spots with radius, $R$, and spacing, $\lambda$. We choose $f_{g}=0.6$ to adjust the average preference of the substrate for PS. The polymer-air surface is modeled as a hard wall. The parameters are matched to the experiment but the qualitative behavior is dictated by the mismatch between the substrate pattern and the bulk morphology. We do not expect it to depend on the specific choice of the interactions.

Monte Carlo (MC) simulations of a bead spring model are performed in the canonical ensemble utilizing the same chain length and segment-substrate interaction, $U_{W}$ with $f_{g}=1$, as the SCMF simulations. Segments of the same type interact via a purely repulsive Lennard-Jones potential with cutoff $r_{c}=\sqrt[6]{2} \sigma . \epsilon / k_{B} T=0.011$ and $\sigma$ denote the energy and length scale of the potential. $\sigma_{\mathrm{PS}-\mathrm{PMMA}}=\sigma$ and $\epsilon_{\mathrm{PS}-\mathrm{PMMA}}=45.45 \epsilon$ are used for interactions between unlike segments corresponding to $\chi N=36.7$. $\Phi N \sigma^{3}=$ 0.7 and $R_{e}=7 \sigma$ yield $\overline{\mathcal{N}}=56$.

In Fig. 1 (top) we present the composition at the top surface as obtained from experiments and the 3D morphology observed in MC and SCMF simulations. For all patterns we observe a long-range ordered and perfectly registered, single quadratically perforated lamella (QPL) at the substrate. The thickness of this QPL sheet is about $L_{0} / 5$. This morphology is not observed in the bulk or on uniform substrates; it is enforced by the substrate interactions, $\Lambda N$, which are sufficiently strong to induce a surface morphology differing in symmetry and characteristic

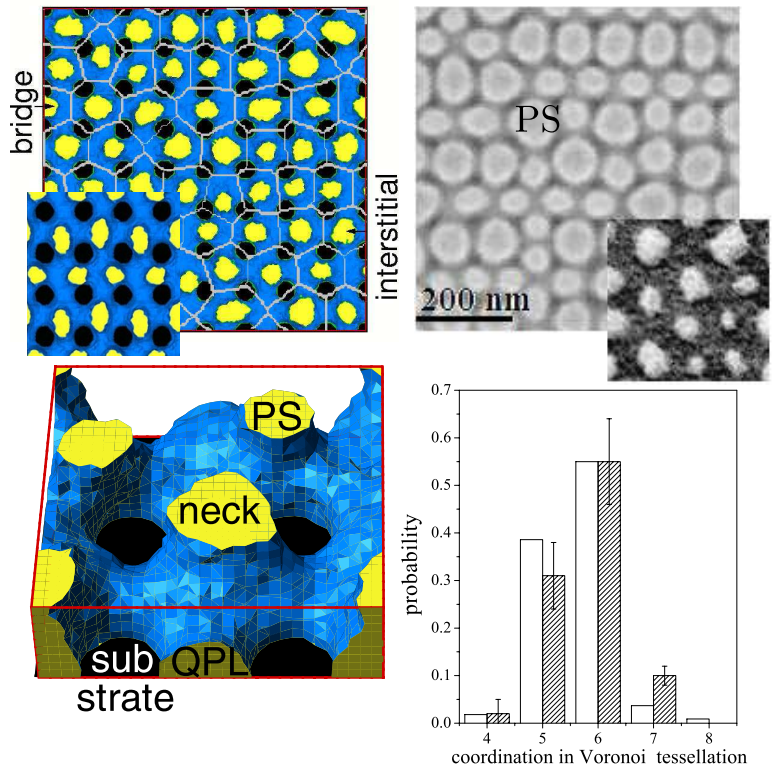

FIG. 1 (color online). Morphology of a copolymer/homopolymer blend film of thickness $D_{0}=0.63 L_{0}$ and lateral dimensions of $9.77 L_{0}$ on a patterned substrate with $\lambda=1.21 L_{0}$ and $R=0.30 L_{0}$. Top left: SCMF simulation showing only the top view of the PS-rich domains (white/yellow online) and the interface between PS and PMMA (dark gray/blue online). Regions on top of the spots are PMMA-rich (transparent) and one looks through to the substrate (black). The position between two PMMA-attracting spots of the substrate is denoted as "bridge," while the position at the center of a plaquette of four spots is denoted "interstitial." The Voronoi tessellation of the necks is indicated by thin white lines. The lower left inset presents the time-averaged morphology in the MC simulations using the same color scheme as for the SCMF simulations. The MC simulation cell is replicated once in each direction. Top right: SEM image of the composition at the film surface. The PS domains are shown in light gray while PMMA-rich surface areas correspond to dark gray regions. Note that the PS domains appear artificially larger in the SEM images so a more accurate view of the domain sizes is provided by an AFM phase image shown as an inset at the lower right corner. Bottom left: 3D view of the morphology in the MC simulations. The QPL and PS-rich necks are indicated. Bottom right: Probability distribution of the coordination (number of edges) of Voronoi cells (cf. top left panel). The "errorbars" of experimental data (shown in gray) characterize the variance of results obtained by analyzing different SEM images of size $9.77 L_{0}$. SCMF simulation results are shown as open bars.

length scale from the bulk structure. We would expect no qualitative change if we increased $\Lambda N$.

On average, the chemically patterned substrate prefers the PS component and its selectivity increases as we decrease the ratio between the spot radius and spacing, $R / \lambda$. The top of the film is PS depleted forming a perforated PMMA-rich sheet [25]. For small values of $R / \lambda$ the perforations consist of necks (short standing cylinders of PS) connecting the QPL underneath to the top of the film giving rise to a bicontinuous structure [26]. 
The position of the PS-rich necks emanating from the QPL is determined by the interplay between their natural size (set by $R_{e}$ ), their preferred packing in the plane parallel to the substrate, and the geometry (size and curvature) of the location on the QPL to which they are connected. Thus the location and shape of the PS-rich domains at the top of the film provide substantial but indirect information about the 3D morphology. Necks that form on bridge positions (cf. Figure 1) in the simulations have a smaller diameter, on average, than those forming at interstitial positions. This partially explains the rather large distribution of neck diameters observed in the experiments. Although the substrate and QPL possess a fourfold symmetry, the Voronoi tessellation presented in Fig. 1 (bottom right) exhibits a large fraction of locally sixfold coordinated necks both in experiments and simulations due to the local packing of necks. Analysis of the orientational correlation functions [16] shows that hexagonal orientations are strong but short ranged, while substrate-induced fourfold orientational correlations are weak but longer ranged [27]. This explains the lack of symmetry between Voronoi polygons with 5 and 7 sides. In comparison to adsorbed monolayers on corrugated substrates, the necks do not exist in the bulk but self-assemble due to substrate interactions and confinement. The film structure is nonuniform perpendicular to the substrate; i.e., it is truly three-dimensional.

In Fig. 2 (top) we increase the ratio $R / \lambda$ but leave the periodicity $\lambda$ unaltered compared to Fig. 1 , and the substrate becomes less preferential to PS, on average. The amount of PS near the top surface of the film increases facilitating the formation of a bulklike lamellar morphology. Indeed, comparing Figs. 1 and 2 (top) we observe that neighboring necks join into portions of standing lamellae that are connected to the QPL at the substrate and orient along the axis of the QPL. Increasing $R$, we decrease the width of the bridge and interstitial positions and all necks are now located on the wider interstitial positions. This alignment of the structures with the axis of the QPL is also observed in experiments.

The joining of necks into short portions of lamellae is also observed in Fig. 2 (bottom), consistent with the increased ratio, $R / \lambda$, as compared to Fig. 1. Although the length scale, $\lambda$, of the substrate pattern substantially exceeds the bulk period, $L_{0}$, the substrate interactions force the formation of a QPL at the substrate. The width of the necks and short lamellar portions on the top, however, remains largely unaltered. The portions of lamellae either run along the diagonal or align parallel with the axis of the underlying square lattice. In the latter case, they bridge the PMMA-rich domains of the QPL. In response to the increase of the dimensions of the QPL, all necks now register with the bridge positions that are narrower than the interstitial ones. The bridge positions form a square lattice whose axes are rotated by $45^{\circ}$ with respect to the QPL and this diagonal orientation of the necks is clearly visible in the experimental results.
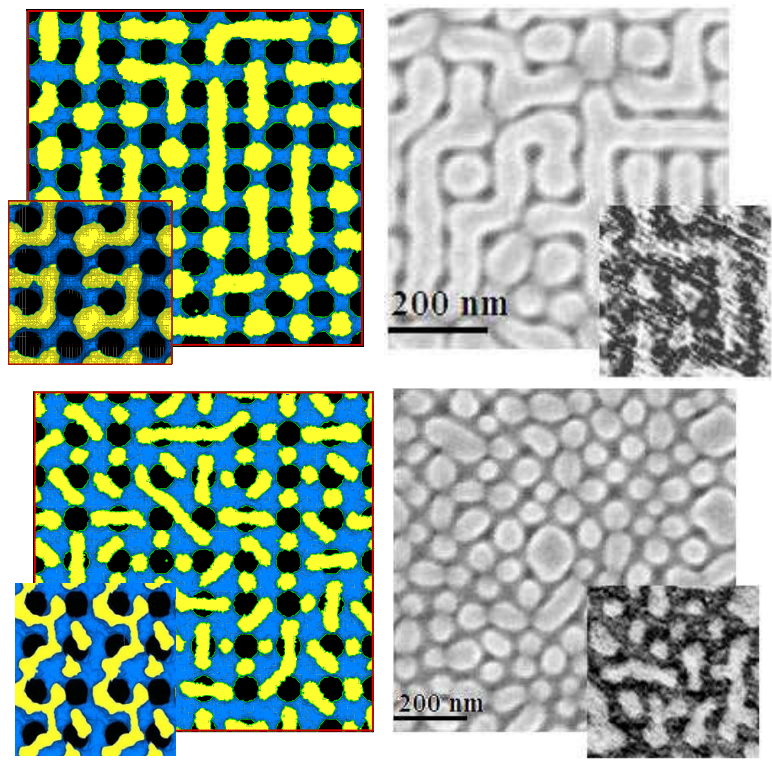

FIG. 2 (color online). Morphologies observed for $\lambda=1.21 L_{0}$, $R=0.41 L_{0}$ (top) and $\lambda=1.64 L_{0}, R=0.51 L_{0}$ (bottom). The lateral dimensions are $9.77 L_{0}$ and $13.2 L_{0}$, respectively. The left panels show SCMF and MC (insets) simulation results. The corresponding SEM and AFM images are shown on the main and inset plates of the panels on the right.

Ultimately the morphology created by reconstruction on a patterned substrate does not alter the morphology at macroscopic distances from the substrate. Thus, in Fig. 3 we explore the morphology of thicker films via SCMF simulations. At twice the film thickness we observe a PS-rich QPL at the substrate followed by alternating PMMA-rich and PS-rich perforated sheets. Even for this thicker film the morphology does not revert to the lamellar bulk morphology at the top surface. The structure in the middle of the film also resembles the surface structure of a film of half the thickness (see Fig. 1).

The prominent features of the thin film morphologies are the bicontinuity of both components and the perfect registration with the substrate pattern. Since the length scale of these morphologies is dictated by the molecular extension it can be tuned over the range of a few tens of nanometers.

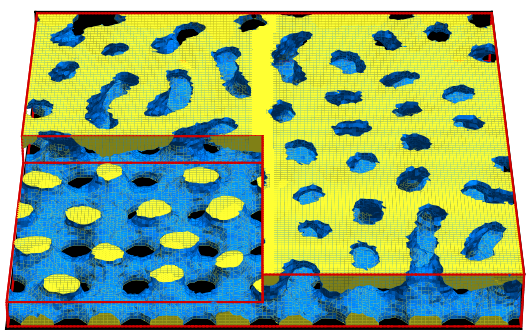

FIG. 3 (color online). "Swiss cheese" morphology formed in the SCMF simulations on the same pattern as in Fig. 1 but for twice the film thickness, $D_{0}=1.27 L_{0}$. In the lower left corner the upper half of the film is removed revealing a morphology similar to Fig. 1. 
Much effort has been directed towards creating bicontinuous morphologies at these dimensions. The large surface to volume ratios that they provide are advantageous for applications in catalysis [28], separation membranes, and filtration [29]. Moreover, they have the potential to exhibit interesting mechanical [30,31] or optical properties [32]. Periodic bicontinuous morphologies with 3D connectivity exist in pure diblock copolymers only in a very limited range of asymmetric molecular architectures and exhibit long-range order. Bicontinuous microemulsions in copolymer-homopolymer blends $[33,34]$ also exist only in a very limited composition range. Our study suggests a robust way of fabricating bicontinuous morphologies using lamellar-forming diblock materials on patterned substrates without needing to fine-tune the block copolymer composition or architecture. The thermodynamically stable [24] bicontinuous structures result from (1) strong preferential interactions between the components of the blend and the surface and (2) the mismatch between the morphology of the blend in the bulk and the geometry and length scale of the chemical surface pattern. The perfect registration of the morphologies with the substrate pattern provides the ability to address the components of the bicontinuous thin film morphologies from the side of the patterned substrate and thereby potentially enables connections to external devices.

This work illustrates the principal concept of fabricating complex 3D structures by directed assembly on lithographically patterned substrates. Currently it is possible to pattern self-assembling materials in two dimensions at the nanoscale with perfection and registration over macroscopic areas. Nanoscale manufacturing capabilities can be significantly enhanced if 3D structures could be created from single 2D templates exploiting the perfection of the lithographic patterning. As in the initial demonstration of this concept to fabricate addressable bicontinuous network structures, a combined experimental and theoretical approach is essential.

We thank F.S. Bates for stimulating discussions. Support by the Semiconductor Research Corporation (SRC) (2005-OC-985), the National Science Foundation through the Nanoscale Science and Engineering Center (DMR-0425880), the Volkswagen foundation, and the John von Neumann Institute for computing is gratefully acknowledged. This work was performed using the facilities and staff at the UW Center for Nanotechnology and the Swiss Light Source. M. P. S. acknowledges additional support from SRC.

*mmueller@theorie.physik.uni-goettingen.de

†nealey@engr.wisc.edu

[1] S. H. Anastasiadis et al., Phys. Rev. Lett. 62, 1852 (1989).

[2] P. Mansky et al., Science 275, 1458 (1997).
[3] M. S. Turner, Phys. Rev. Lett. 69, 1788 (1992).

[4] A. Knoll et al., Phys. Rev. Lett. 89, 035501 (2002).

[5] N. Rehse et al., Phys. Rev. Lett. 87, 035505 (2001).

[6] K. Huang and A.C. Balazs, Phys. Rev. Lett. 66, 620 (1991).

[7] A. Halperin, J.-U. Sommer, and M. Daoud, Europhys. Lett. 29, 297 (1995).

[8] G. G. Pereira and D. R. M. Williams, Phys. Rev. Lett. 80, 2849 (1998).

[9] L. Rockford et al., Phys. Rev. Lett. 82, 2602 (1999).

[10] Q. Wang et al., J. Chem. Phys. 112, 9996 (2000).

[11] Y. Tsori and D. Andelman, Europhys. Lett. 53, 722 (2001); Interface Sci. 11, 259 (2003).

[12] S. Stepanow and A. A. Fedorenko, Europhys. Lett. 58, 368 (2002).

[13] S. O. Kim et al., Nature (London) 424, 411 (2003).

[14] E. W. Edwards et al., Adv. Mater. 16, 1315 (2004).

[15] M. P. Stoykovich et al., Science 308, 1442 (2005).

[16] D. R. Nelson and B. I. Halperin, Phys. Rev. B 19, 2457 (1979).

[17] H. H. Solak et al., Microelectron. Eng. 67-68, 56 (2003).

[18] H. H. Solak et al., J. Vac. Sci. Technol. B 20, 2844 (2002).

[19] The symmetric ternary blend behaves very similarly to a pure lamellar-forming block copolymer [13-15]. However, experiments using the pure copolymer with $L_{0}^{\text {pure }}=49 \mathrm{~nm}$ are precluded due to difficulties in patterning at these smaller length scales. If we increased $N$ to achieve $L_{0}^{\text {pure }}=70 \mathrm{~nm}$ the relaxation times would be prohibitively long.

[20] M. Müller and G. D. Smith, J. Polym. Sci., Part B: Polym. Phys. 43, 934 (2005).

[21] G.H. Fredrickson, V. Ganesan, and F. Drolet, Macromolecules 35, 16 (2002).

[22] T. P. Russell, R. P. Hjelm, and P. A. Seeger, Macromolecules 23, 890 (1990).

[23] M. Sferrazza et al., Phys. Rev. Lett. 78, 3693 (1997).

[24] For selected cases we have verified that independent simulation runs yield similar results and we occasionally observe that necks disappear and re-form during a simulation run. Thus the morphologies are equilibrium structures.

[25] Unlike the hexagonally perforated lamellar morphology [D. A. Hajduk et al., Macromolecules 30, 3788 (1997)] of asymmetric diblock copolymers, both phases of our symmetric blend are continuous.

[26] Necks are reminiscent of the surface morphology in cylinder-forming ABA triblock thin films on uniform substrates [M. Konrad et al., Macromolecules 33, 5518 (2000)].

[27] K. Ch. Daoulas et al. (to be published).

[28] C. Park, J. Yoon, and E. L. Thomas, Polymer 44, 6725 (2003).

[29] P. F. W. Simon et al., Chem. Mater. 13, 3464 (2001).

[30] E. W. Cochran and F. S. Bates, Phys. Rev. Lett. 93, 087802 (2004).

[31] T. H. Epps et al., Macromolecules 37, 7085 (2004).

[32] A. C. Edrington et al., Adv. Mater. 13, 421 (2001).

[33] F. S. Bates et al., Phys. Rev. Lett. 79, 849 (1997).

[34] M. Müller and M. Schick, J. Chem. Phys. 105, 8885 (1996). 